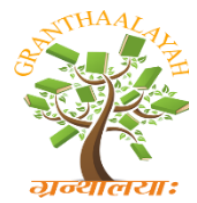

\author{
INTERNATIONAL JOURNAL OF RESEARCH - \\ GRANTHAALAYAH \\ A knowledge Repository
}

Science

\title{
PREVALENCE OF TICK AND MANGE MITES' INFESTATION IN GOATS IN BENATSEMAY DISTRICT OF SOUTH OMO ZONE, ETHIOPIA
}

\author{
Dr Seifu Lemma ${ }^{1}$, Alemayeh Redii ${ }^{1}$ \\ ${ }^{1}$ Jinka Animal Health Laboratory Centre, P.O. Box 62, Jinka Ethiopia
}

\begin{abstract}
Across sectional, study of tick was conducted to identify types of tick species and determine the Prevalence of tick and mange mites infestation in small ruminants in Benatsemayworeda, South Omozone, Southern Nation Nationality of Ethiopia. Collection and identification of the ticks and mange mites were undertaken from June 6----- /15/2019. The overall prevalence of tick and mange mites infestation in the study area was $172(40.66 \%)$ All visible individual adult ticks were collected from the body of 172 goats. The prevalence of tickand mange mites infestation in goats was found to be $(40.66 \%)$. In this study, one/ species of ticks, which grouped under one genus, was identified. The most abundant species found in this study were Rhipicephalus/pulchellus, eversi, simus, B(decoloratus) and psoroptics mites.
\end{abstract}

Keywords: Tick; Mange-Mites; Benatsemay.

Cite This Article: Dr Seifu Lemma, and Alemayeh Redii. (2019). "PREVALENCE OF TICK AND MANGE MITES' INFESTATION IN GOATS IN BENATSEMAY DISTRICT OF SOUTH OMO ZONE, ETHIOPIA." International Journal of Research - Granthaalayah, 7(8), 111-118. https://doi.org/10.29121/granthaalayah.v7.i8.2019.644.

\section{Introduction}

The livestock population of Ethiopia is above 41 million cattle, 14.3 milliosheep and 13 million goats (CSA, 2004) Ethiopia's resources of cattle, sheep and goats ranks first, third and second respectively in Africa (Leach,1998). Because of this huge potential, Ethiopia is capable of supplying 16-18million of skins and hides per year for the tenneries within the country. The Ethiopian skins and hides, especially the sheep skin is known in the world for the production of the finest leather in terms of its fine grain and compact structure. The quality wet-blue goat skins are known as "Bati-genuine" and they have also a good reputation in the international market and receive the biggest attraction for the number of leather producing companies in the world (Mulat Abegaz, 1999)

Small ruminants constitute about $30 \%$ of the total livestock population of the country and important contributors to food production in Ethiopia providing $35 \%$ of meat, $14 \%$ of milk 
consumptions and accounts for $40 \%$ of cash income and $19 \%$ of the use household consumptions (Bayou, 1998; Yacobetal, 2008). Small ruminants are considered as investments and insurance to provide income food during seasons of crop failure and to meet seasonal purchase such as improved seed fertility and medicine for rural household. They have essential attributes like fertilizer, short generation time interval and adaptation even in harsh environment (Asfaw, 1997). The exportation of skin and hides is the largest foreign exchange earners in the country. Yet as many as one quarter to one -third of all the skins processed at tanneries are unsuitable for export due to various defects (KassaBayuet al., 1998). The leather industry sector is one of the fourth growing economic sectors in the country. However, this sector of trade and the country as whole lost revenue due to a decline in quality and fall in export prices (Pittards, 1999; NBE, 2000). Up to $65 \%$ of these defects occur in the pre slaughter stage of production while the animal is still alive. A considerable portion of these pre slaughter defects are due to skin diseases caused by parasites (KassaBayu et al., 1998).

Ectopararasites commonly ticks, mites, lice and ked are important parasites because of their disease transmission, blood feeding habit and skin damage in most of the live stock population (CSA, 2004). Losses from tick damage to hides and skin were claimed to be about one million Ethiopian birr per annum, but are likely to be much higher (SileshiMekonnen, 1992).

Mange is a collective name for allergic dermatitis caused by ectoparasitic infestations by mites that are obligate parasites and spread from animal to animal by direct contact. Mange usually appears as a skin conditions associated with irritation and scratching that leads to inflammation, exudation, crusts and scabs forming on skin. Untreated mange leads to thickening of skin and loss of condition of the animal. The disease is often seen in animals in generally poor condition and during the winter season and characterized by a variety of clinical signs depends on the species (Blood and Radostitis, 1989; Dorny et al., 1994; Lusilukaet al., 1995; Pangui, 1994; Radostitis et al., 2007; Taylor et al., 2007; and Wall and Shearer, 1997).

Furthermore, ectoparasitic mites cause mange in a wide range of domestic and wild vertebrates hosts including man. A considerable economic negative impact associated with mange infection is due to pesticide prices, extensive skin damage and morbidity and mortality of the infested animals (Osman et al., 2006).

Despite enormous efforts carried out by government veterinary personnel, community animal health workers and private practitioners in the woreda to treat and control tick and mange mite infestations in the woreda, there are persistent complains over those problems regarding their effect on health and productivity of the livestock.

The survey carried out by Sodo regional veterinary laboratory in 2007 showed overall prevalence of $88.54 \%$ in Bana tsemay woreda of south Omo zone;

Again the results of rapid assessments of ecto-parasites in sheep and goats in 12 woredas that was conducted by Sodo and Mizan regional veterinary laboratories in 2008 showed significantly high prevalence of different parasites. For example: $46.05 \%$ prevalence of mange in Hamer woreda of South Omo zone in goats. 
The objectives of this study were thus to assess the levels of prevalence of tickinfestation and comparing the past with the present in the district and make inference on the effectiveness of treatment and control efforts made in the meantime and conclude possible options.

\section{Materials and Methods}

\section{Description of the Study Area}

The study was conducted in Benatsemay district of South Omo Zone located at $5^{0} .01--5^{0} .73$ ' N

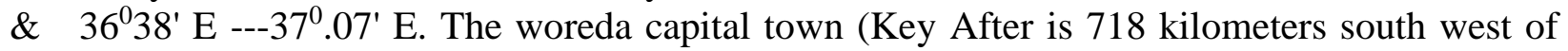
Addis Aaba (the SNNP Regional Capital) and $42 \mathrm{~km}$ to the south east of Jinka (the South Omo Zonal Capital). The total area of the woreda is 2,922.8 sq. kilo meters. The altitude of the woreda ranges from $<500$ to 2,500 meters above sea level and has both the hot arid as well as the midland (woina dega) types of climate. It has two main rainy seasons March to May and from October to December; the area also occasionally enjoys the July-August rainfall that is common to the central highlands of Ethiopia.

The human population of the woreda is both sex 62,825 male 31,788 female 31,037 . The woreda has sheep and goat populations of 155,485 and 421,738 respectively.

\section{The Survey Design}

Single round visiting for clinical examination of shoats was designed in Benatsemayworeda. Three Kebeles were selected from the woreda for the survey purpose by trying to diversify the ecology and geographic location of the kebeles.

The field program was arranged by Jinka laboratory with the aim of disease surveillance and investigation in BenaTesmayworeda.

The field/survey/program was from June 6-15/2011 E.C

\section{The Samples}

A total of 423 goats were the sample animals selected randomly from the 3 kebeles of BenaTesmay woreda. The field observation findings were recorded and specimens of external parasites collected for further identification.

\section{Clinical and Laboratory Investigation}

All visible attached adult ticks were collected carefully and gently removed exerting a horizontal pull to the body surface by rotating the tick not to damage the host by the tick mouth parts and then the collected ticks were preserved in properly labeled plastic container containing $70 \%$ ethanol. The bottle was labeled with date, place, sex, age and site of the body and then transported to Jinka Veterinary Regional Laboratory for parasitological analysis. The collected ticks from each container was placed onto Petri dishes and examined under stereomicroscope to identify the species using tick identification keys described by [Walker et. al,2003]; [Fischer et al 1989]. Briefly, the main identification features of the ticks are color, size, and shape of mouth parts, Scutum, anal groove, festoon, punctuation and legs [Onkeelo et. al, 2006]. 


\section{Questionnaire Survey and Review of Secondary Data}

The following questions were interviewed with the livestock owners:

1) What are the major external parasites causing problem to your shoats?

2) Grazing habits/modalities, how far do shoats and cattle share the same grazing and watering points?

3) Traditional and modern methods of tick control currently in use and the gaps observed on applying those methods.

Table 1: Observation of the levels tick and mange mites infestation of the past Result (Benatsemayworeda) 2017

\begin{tabular}{|c|c|c|c|c|c|c|c|c|c|c|c|c|}
\hline \multirow[t]{3}{*}{ Woreda } & \multicolumn{10}{|c|}{ No and \% of animals infested with each type of external parasites } & \multirow{2}{*}{\multicolumn{2}{|c|}{$\begin{array}{l}\text { Overall*** } \\
\text { infestations }\end{array}$}} \\
\hline & \multirow[t]{2}{*}{ Species } & \multirow{2}{*}{$\begin{array}{c}\text { No. } \\
\text { examined }\end{array}$} & \multicolumn{2}{|c|}{ Mange mites } & \multicolumn{2}{|c|}{ Ticks } & \multicolumn{2}{|c|}{ Lice } & \multicolumn{2}{|c|}{ Fleas } & & \\
\hline & & & $+\mathrm{ve}$ & \%ve & +ve & $\%$ ve & +ve & \%ve & +ve & $\%$ ve & $+\mathrm{ve}$ & \%ve \\
\hline \multirow[t]{3}{*}{ Benstsemay } & Sheep & 37 & 3 & $8.11 \%$ & 26 & $70.27 \%$ & 0 & $0.00 \%$ & 0 & $0.00 \%$ & & $0.00 \%$ \\
\hline & Goats & 209 & 46 & $22.01 \%$ & 159 & $76.08 \%$ & 0 & $0.00 \%$ & 0 & $0.00 \%$ & & $0.00 \%$ \\
\hline & Total & 246 & 49 & $19.92 \%$ & 185 & $75.20 \%$ & 0 & $0.00 \%$ & 0 & $0.00 \%$ & 185 & $75.20 \%$ \\
\hline
\end{tabular}

Over all prevalence $73.145 \%$

Result of (2017)

\section{Results and discussion}

\section{Results June 2019}

What are the major external parasites causing problem to your shoats?

1) It was reported in all the kebeles by all the respondents that ticks and mange mites are still a major problem to the sheep and goats in the woreda. The do harm by sucking blood, transmitting/predisposing to diseases and damaging organs, reducing the quality of skin, and through costs incurred to treat and control the infestations.

Grazing habits/modalities, how far do shoats and cattle share the same grazing and watering poits?

2) $92 \%$ of the respondents have declared that the shoats and cattle often share similar grazing areas and watering points during the rainy season.

3) Traditional and modern methods of tick control currently in use and the gaps observed on applying those methods.

- Burning of dry feces in barns

- Controlled burning of grass lands (but now not common due to shortage of grazing pasture) 
Table 2: Observation of the levels tick and mange mites' infestation in Bena tesamyworeda June 2019

DISEASE SURVEILLANCE Dep. Parasitology wereda Bena tsemay kebele Alduba

\begin{tabular}{|c|c|c|c|c|c|c|}
\hline $\begin{array}{l}\text { Customer } \\
\text { code }\end{array}$ & $\begin{array}{l}\text { Lab } \\
\text { code }\end{array}$ & Species & $\begin{array}{c}\text { Disease } \\
\text { type }\end{array}$ & $\begin{array}{c}\text { Sample } \\
\text { type }\end{array}$ & Test type & Result \\
\hline 1 & T01 & Caprine & $\begin{array}{l}\text { External } \\
\text { parasite }\end{array}$ & Ticks & $\begin{array}{l}\text { Morphological } \\
\text { identification }\end{array}$ & $\begin{array}{l}\text { R.(eversi, puluchilis,simus) } \\
\text { B.(decoloratus) }\end{array}$ \\
\hline 2 & T02 & Caprine & $\begin{array}{l}\text { External } \\
\text { parasite }\end{array}$ & Ticks & $\begin{array}{l}\text { Morphological } \\
\text { identification }\end{array}$ & $\begin{array}{l}\text { R.(eversi, puluchilis,simus) } \\
\text { B.(decoloratus) }\end{array}$ \\
\hline 3 & T03 & Caprine & $\begin{array}{l}\text { External } \\
\text { parasite }\end{array}$ & Ticks & $\begin{array}{l}\text { Morphological } \\
\text { identification }\end{array}$ & $\begin{array}{l}\text { R.(eversi, puluchilis,simus) } \\
\text { B.(decoloratus) }\end{array}$ \\
\hline 4 & T04 & Caprine & $\begin{array}{l}\text { External } \\
\text { parasite }\end{array}$ & Ticks & $\begin{array}{l}\text { Morphological } \\
\text { identification }\end{array}$ & $\begin{array}{l}\text { R.(eversi, puluchilis,simus) } \\
\text { B.(decoloratus) }\end{array}$ \\
\hline 5 & T05 & Caprine & $\begin{array}{l}\text { External } \\
\text { parasite }\end{array}$ & Ticks & $\begin{array}{l}\text { Morphological } \\
\text { identification }\end{array}$ & $\begin{array}{l}\text { R.(eversi, puluchilis,simus) } \\
\text { B.(decoloratus) }\end{array}$ \\
\hline 6 & T06 & Caprine & $\begin{array}{l}\text { External } \\
\text { parasite }\end{array}$ & Ticks & $\begin{array}{l}\text { Morphological } \\
\text { identification }\end{array}$ & $\begin{array}{l}\text { R.(eversi, puluchilis,simus) } \\
\text { B.(decoloratus) }\end{array}$ \\
\hline 7 & T07 & Caprine & $\begin{array}{l}\text { External } \\
\text { parasite }\end{array}$ & Ticks & $\begin{array}{l}\text { Morphological } \\
\text { identification }\end{array}$ & $\begin{array}{l}\text { R.(eversi, puluchilis,simus) } \\
\text { B.(decoloratus) }\end{array}$ \\
\hline 8 & T08 & Caprine & $\begin{array}{l}\text { External } \\
\text { parasite }\end{array}$ & Mites & $\begin{array}{l}\text { Morphological } \\
\text { identification }\end{array}$ & Psorotic mites \\
\hline 9 & T09 & Caprine & $\begin{array}{l}\text { External } \\
\text { parasite }\end{array}$ & Mites & $\begin{array}{l}\text { Morphological } \\
\text { identification }\end{array}$ & $\begin{array}{l}\text { Psoroptic mites (in less } \\
\text { infestation) }\end{array}$ \\
\hline 10 & T10 & Caprine & $\begin{array}{l}\text { External } \\
\text { parasite }\end{array}$ & Mites & $\begin{array}{l}\text { Morphological } \\
\text { identification }\end{array}$ & $\begin{array}{l}\text { Psoroptic mites (in less } \\
\text { infestation) }\end{array}$ \\
\hline 11 & T11 & Caprine & $\begin{array}{l}\text { External } \\
\text { parasite }\end{array}$ & Mites & $\begin{array}{l}\text { Morphological } \\
\text { identification }\end{array}$ & $\begin{array}{l}\text { Psoroptic mites (in less } \\
\text { infestation) }\end{array}$ \\
\hline 12 & T12 & Caprine & $\begin{array}{l}\text { External } \\
\text { parasite }\end{array}$ & Mites & $\begin{array}{l}\text { Morphological } \\
\text { identification }\end{array}$ & $\begin{array}{l}\text { Psoroptic mites (in less } \\
\text { infestation) }\end{array}$ \\
\hline 13 & T13 & Caprine & $\begin{array}{l}\text { External } \\
\text { parasite }\end{array}$ & Mites & $\begin{array}{l}\text { Morphological } \\
\text { identification }\end{array}$ & $\begin{array}{l}\text { Psoroptic mites (in less } \\
\text { infestation) }\end{array}$ \\
\hline 14 & T14 & Caprine & $\begin{array}{l}\text { External } \\
\text { parasite }\end{array}$ & Mites & $\begin{array}{l}\text { Morphological } \\
\text { identification }\end{array}$ & $\begin{array}{l}\text { Psoroptic mites (in less } \\
\text { infestation) }\end{array}$ \\
\hline
\end{tabular}

Table 3: DISEASE SURVEILLANCE Dep. Parasitology wereda Bena tsemay kebele uffi , June 2019

\begin{tabular}{|l|l|l|l|l|l|l|}
\hline $\begin{array}{c}\text { Customer } \\
\text { code }\end{array}$ & $\begin{array}{l}\text { Lab } \\
\text { code }\end{array}$ & Species & $\begin{array}{l}\text { Disease } \\
\text { type }\end{array}$ & $\begin{array}{l}\text { Sample } \\
\text { type }\end{array}$ & \multicolumn{1}{|c|}{ Test type } & \multicolumn{1}{|c|}{ Result } \\
\hline 1 & T01 & Caprine & $\begin{array}{l}\text { External } \\
\text { parasite }\end{array}$ & Ticks & $\begin{array}{l}\text { Morphological } \\
\text { identification }\end{array}$ & $\begin{array}{l}\text { R.(eversi, puluchilis,simus) } \\
\text { B.(decoloratus) }\end{array}$ \\
\hline 2 & T02 & Caprine & $\begin{array}{l}\text { External } \\
\text { parasite }\end{array}$ & Ticks & $\begin{array}{l}\text { Morphological } \\
\text { identification }\end{array}$ & $\begin{array}{l}\text { R.(eversi, puluchilis,simus) } \\
\text { B.(decoloratus) }\end{array}$ \\
\hline 3 & T03 & Caprine & $\begin{array}{l}\text { External } \\
\text { parasite }\end{array}$ & Ticks & $\begin{array}{l}\text { Morphological } \\
\text { identification }\end{array}$ & $\begin{array}{l}\text { R.(eversi, puluchilis,simus) } \\
\text { B.(decoloratus) }\end{array}$ \\
\hline 4 & T04 & Caprine & $\begin{array}{l}\text { External } \\
\text { parasite }\end{array}$ & Ticks & $\begin{array}{l}\text { Morphological } \\
\text { identification }\end{array}$ & $\begin{array}{l}\text { R.(eversi, puluchilis,simus) } \\
\text { B.(decoloratus) }\end{array}$ \\
\hline 5 & T05 & Caprine & $\begin{array}{l}\text { External } \\
\text { parasite }\end{array}$ & Ticks & $\begin{array}{l}\text { Morphological } \\
\text { identification }\end{array}$ & $\begin{array}{l}\text { R.(eversi, puluchilis,simus) } \\
\text { B.(decoloratus) }\end{array}$ \\
\hline
\end{tabular}




\begin{tabular}{|l|l|l|l|l|l|lll|}
\hline 6 & T06 & Caprine & $\begin{array}{l}\text { External } \\
\text { parasite }\end{array}$ & Ticks & $\begin{array}{l}\text { Morphological } \\
\text { identification }\end{array}$ & $\begin{array}{l}\text { R.(eversi, puluchilis,simus) } \\
\text { B.(decoloratus) }\end{array}$ \\
\hline 7 & T07 & Caprine & $\begin{array}{l}\text { External } \\
\text { parasite }\end{array}$ & Ticks & $\begin{array}{l}\text { Morphological } \\
\text { identification }\end{array}$ & $\begin{array}{l}\text { R.(eversi, puluchilis,simus) } \\
\text { B.(decoloratus) }\end{array}$ & \\
\hline 8 & T08 & Caprine & $\begin{array}{l}\text { External } \\
\text { parasite }\end{array}$ & Mites & $\begin{array}{l}\text { Morphological } \\
\text { identification }\end{array}$ & Psorotic mites & \\
\hline 9 & T09 & Caprine & $\begin{array}{l}\text { External } \\
\text { parasite }\end{array}$ & Mites & $\begin{array}{l}\text { Morphological } \\
\text { identification }\end{array}$ & $\begin{array}{l}\text { Psoroptic mites } \\
\text { infestation) }\end{array}$ & (in less \\
\hline 10 & T10 & Caprine & $\begin{array}{l}\text { External } \\
\text { parasite }\end{array}$ & Mites & $\begin{array}{l}\text { Morphological } \\
\text { identification }\end{array}$ & $\begin{array}{l}\text { Psoroptic mites } \\
\text { infestation) }\end{array}$ & (in less \\
\hline 11 & T11 & Caprine & $\begin{array}{l}\text { External } \\
\text { parasite }\end{array}$ & Mites & $\begin{array}{l}\text { Morphological } \\
\text { identification }\end{array}$ & $\begin{array}{l}\text { Psoroptic mites } \\
\text { infestation) }\end{array}$ & (in less \\
\hline 12 & T12 & Caprine & $\begin{array}{l}\text { External } \\
\text { parasite }\end{array}$ & Mites & $\begin{array}{l}\text { Morphological } \\
\text { identification }\end{array}$ & $\begin{array}{l}\text { Psoroptic mites } \\
\text { infestation) }\end{array}$ (in less \\
\hline 13 & T13 & Caprine & $\begin{array}{l}\text { External } \\
\text { parasite }\end{array}$ & Mites & $\begin{array}{l}\text { Morphological } \\
\text { identification }\end{array}$ & $\begin{array}{l}\text { Psoroptic mites } \\
\text { infestation) }\end{array}$ (in less \\
\hline 14 & T14 & Caprine & $\begin{array}{l}\text { External } \\
\text { parasite }\end{array}$ & Mites & $\begin{array}{l}\text { Morphological } \\
\text { identification }\end{array}$ & $\begin{array}{l}\text { Psoroptic mites } \\
\text { infestation) }\end{array}$ & (in less \\
\hline
\end{tabular}

Table 4: DISEASE SURVEILLANCE Dep. Parasitology weredaBenatsemaykebeleKako, June

\begin{tabular}{|c|c|c|c|c|c|c|}
\hline \multicolumn{7}{|c|}{2019} \\
\hline $\begin{array}{l}\text { Customer } \\
\text { code }\end{array}$ & $\begin{array}{l}\text { Lab } \\
\text { code }\end{array}$ & Species & $\begin{array}{l}\text { Disease } \\
\text { type }\end{array}$ & $\begin{array}{c}\text { Sample } \\
\text { type }\end{array}$ & Test type & Result \\
\hline 1 & T01 & Caprine & $\begin{array}{l}\text { External } \\
\text { parasite }\end{array}$ & Ticks & $\begin{array}{l}\text { Morphological } \\
\text { identification }\end{array}$ & $\begin{array}{l}\text { R.(eversi, puluchilis,simus) } \\
\text { B.(decoloratus) }\end{array}$ \\
\hline 2 & T02 & Caprine & $\begin{array}{l}\text { External } \\
\text { parasite }\end{array}$ & Ticks & $\begin{array}{l}\text { Morphological } \\
\text { identification }\end{array}$ & $\begin{array}{l}\text { R.(eversi, puluchilis,simus) } \\
\text { B.(decoloratus) }\end{array}$ \\
\hline 3 & T03 & Caprine & $\begin{array}{l}\text { External } \\
\text { parasite }\end{array}$ & Ticks & $\begin{array}{l}\text { Morphological } \\
\text { identification }\end{array}$ & $\begin{array}{l}\text { R.(eversi, puluchilis,simus) } \\
\text { B.(decoloratus) }\end{array}$ \\
\hline 4 & T04 & Caprine & $\begin{array}{l}\text { External } \\
\text { parasite }\end{array}$ & Ticks & $\begin{array}{l}\text { Morphological } \\
\text { identification }\end{array}$ & $\begin{array}{l}\text { R.(eversi, puluchilis,simus) } \\
\text { B.(decoloratus) }\end{array}$ \\
\hline 5 & T05 & Caprine & $\begin{array}{l}\text { External } \\
\text { parasite }\end{array}$ & Ticks & $\begin{array}{l}\text { Morphological } \\
\text { identification }\end{array}$ & $\begin{array}{l}\text { R.(eversi, puluchilis,simus) } \\
\text { B.(decoloratus) }\end{array}$ \\
\hline 6 & T06 & Caprine & $\begin{array}{l}\text { External } \\
\text { parasite }\end{array}$ & Ticks & $\begin{array}{l}\text { Morphological } \\
\text { identification }\end{array}$ & $\begin{array}{l}\text { R.(eversi, puluchilis,simus) } \\
\text { B.(decoloratus) }\end{array}$ \\
\hline 7 & T07 & Caprine & $\begin{array}{l}\text { External } \\
\text { parasite }\end{array}$ & Ticks & $\begin{array}{l}\text { Morphological } \\
\text { identification }\end{array}$ & $\begin{array}{l}\text { R.(eversi, puluchilis,simus) } \\
\text { B.(decoloratus) }\end{array}$ \\
\hline 8 & T08 & Caprine & $\begin{array}{l}\text { External } \\
\text { parasite }\end{array}$ & Mites & $\begin{array}{l}\text { Morphological } \\
\text { identification }\end{array}$ & Psorotic mites \\
\hline 9 & T09 & Caprine & $\begin{array}{l}\text { External } \\
\text { parasite }\end{array}$ & Mites & $\begin{array}{l}\text { Morphological } \\
\text { identification }\end{array}$ & $\begin{array}{l}\text { Psoroptic mites (in less } \\
\text { infestation) }\end{array}$ \\
\hline 10 & T10 & Caprine & $\begin{array}{l}\text { External } \\
\text { parasite }\end{array}$ & Mites & $\begin{array}{l}\text { Morphological } \\
\text { identification }\end{array}$ & $\begin{array}{l}\text { Psoroptic mites (in less } \\
\text { infestation) }\end{array}$ \\
\hline 11 & T11 & Caprine & $\begin{array}{l}\text { External } \\
\text { parasite }\end{array}$ & Mites & $\begin{array}{l}\text { Morphological } \\
\text { identification }\end{array}$ & $\begin{array}{l}\text { Psoroptic mites (in less } \\
\text { infestation) }\end{array}$ \\
\hline 12 & T12 & Caprine & $\begin{array}{l}\text { External } \\
\text { parasite }\end{array}$ & Mites & $\begin{array}{l}\text { Morphological } \\
\text { identification }\end{array}$ & $\begin{array}{l}\text { Psoroptic mites (in less } \\
\text { infestation) }\end{array}$ \\
\hline 13 & T13 & Caprine & $\begin{array}{l}\text { External } \\
\text { parasite }\end{array}$ & Mites & $\begin{array}{l}\text { Morphological } \\
\text { identification }\end{array}$ & $\begin{array}{l}\text { Psoroptic mites (in less } \\
\text { infestation) }\end{array}$ \\
\hline
\end{tabular}




\begin{tabular}{|l|l|l|l|l|l|l|}
14 & T14 & Caprine & $\begin{array}{l}\text { External } \\
\text { parasite }\end{array}$ & Mites & $\begin{array}{l}\text { Morphological } \\
\text { identification }\end{array}$ & $\begin{array}{l}\text { Psoroptic mites (in less } \\
\text { infestation) }\end{array}$ \\
\hline
\end{tabular}

Table 5: Observation of the levels tick and mange mites infestation from June 2019

\begin{tabular}{|c|c|c|c|c|c|}
\hline $\begin{array}{c}\text { No. of animal } \\
\text { examined }\end{array}$ & Ticks & Prevalence\% & $\begin{array}{c}\text { Mange } \\
\text { mites }\end{array}$ & Prevalence\% & $\begin{array}{c}\text { Total prevalence } \\
\%\end{array}$ \\
\hline 72 & 48 & 66.66 & & & 66.66 \\
\hline 223 & 59 & 26.46 & 44 & 19.73 & 46.19 \\
\hline 128 & 12 & 9.38 & 9 & 7.03 & 16.41 \\
\hline 423 & 119 & 28.13 & 53 & 12.52 & 40.66 \\
\hline
\end{tabular}

\section{The Study Animals}

Visiting for clinical examination on of 423 goats was carried out in three kebeles were selected randomly and 423 goats were subject to clinical examination. Among the examined goat $119(28.13)$ tick and 53(12.52\%) mange mites were found to have been less infested.

\section{Discussion}

The current study revealed that tick infestation is still widespread and most significant external Parasites of small ruminants in the study area. In this study, 423 small ruminants were examined and a total of 172visible adult ticks and mange were collected from the body of 172 goats. The distribution and abundance of tick species infesting small ruminants in Ethiopia are vary from area to area. In the present study $172(40.66 \%)$ of small ruminants were found to be infested by two species of ticks and mange. Lower prevalence of tick and mange infestation in the study area was observed. This finding was comparable with the previous investigation conducted by the prevalence of tick infestation in the current study was lower than previous works conducted [1] in selected districts of Tigray region, Ethiopia with prevalence of 58.8\% (goats) [8]. In and around Wolaita Soddo, Southern Ethiopia with the prevalence of $18.6 \%$ (goats) and $31.8 \%$ (sheep) and [5]. in North East Ethiopia with prevalence of 3.4\% (goats) and 22.2\% (sheep, however, it is lower than the last study of Jinka and NADIC laboratory which shows Over all prevalence $73.145 \%$

The difference in the Prevalence might be due to the geographical difference, and season of the study period, frequent exposure to the same communal grazing land that favored the frequent contact and management of animals, also the awareness creation to the pastoralist about the application of accaricide.

Moreover, stress of production as pregnancy and lactation could have made the female animals more susceptible to infection. The principal tick species infesting small ruminants in the study area comprise Boophilus decoloratus, Rhipicephalus evertsi, Rhipicephalus pulchellus and Rhipicephalus simus, were less frequent and lower infestation with psoroptic mange (2]

Fully engorged female tick drops off to the ground to lay eggs while male tend to remain on the host up to several months to continue feeding and mating with other females on the host before dropping off [6].The females of B. decoloratus; outnumbered males in this study probably due to small size of male, which may not be seen during collection. 
Ticks and mange's are the most important ecto parasites of goats in tropical and sub-tropical areas, and are responsible for severe economic losses both through the direct effects of blood sucking and indirectly as vectors of pathogens and toxins. This study also confirms that ticks and mange still big concern of ecto parasites and which pose significant challenges to small ruminant production and health of the study area.

\section{Conclusion and Recommendations}

In the study area, the following species of ticks and mange were identified: Rhipicephalusevertsi Boophilusde coloratus, Rhipicephalus pulchellus Rhipicephalus simus and Boophilus decoloratus, and mange-psoroptic.

Therefore, better control could be attained if an emphasis is being input on accaricide small ruminants as per the current finding. Further studies on epidemiological occurrence and influence of environment, tick related risk factors, and of tick-borne diseases, as well as related factors should be conducted in order to set appropriate measures.

\section{References}

[1] Abebe, R., Tatek, M., Megersa, B., and Sheferewu, D. (2011): Prevalence of Small Ruminant Ectoparasites and Associated Risk Factors in Selected Districts of Tigray Region, Ethiopia. Global Veterinaria.7 (5): 433-437.

[2] Abunna, F., Kasasa, B., Shelima, B., Megersa, B., Regassa, A., and Amenu, K. (2007): Survey of tick infestation in small ruminants of Miesso district, West Harargie, Oromia Region, Ethiopia. Tropical Animal Health and Production. 41: 969-972.

[3] Fischer, M., and Ralph Say, R. (1989): Manual of Tropical Veterinary Parasitology. CAB International, Wallingford, U.K. Part III by P.-C.Morelon Tick-borne diseases of Livestock in Africa has information on biology and identification. Guide to identification of species. Bioscience Report. Pp: 1-221.

[4] Onkello-Onen, J., Hassan, S.M., and Essuman, S. (2006): Taxonomy of Africa tick: An identification Manual. International Center for Insect Physiology and Ecology Press, Nairobi, Kenya. Pp: 1-124.

[5] Sertse, T., and Wosene, A. (2007): A study on ectoparasites of sheep and goats in eastern part of Amhara region, Northeast Ethiopia. Small Ruminant Research. 69:62-67.

[6] Solomon, G., Night, M., and Kaaya, GP. (2001a): Seasonal variation of ticks on calves at Sabetha, Western Shewa zone. Ethiopia Vet. J. 7(1\&2):17-30.

[7] Walker, A., Bovattour, J., Camicas, I., Horak and Latif, A. (2003): Ticks of domestic animals in Africa: A guide to identification of species. Bio Science.Report. Pp: 1-122. Wallingford, UK. Pp: 440-485.

[8] Yacob, H.T., Yalow, A.T., and Dink, A. A. (2008): Ectoparasites prevalence in sheep and in goats in and around WolaitaSoddo, Southern Ethiopia. American-Eurasian Journal of Agriculture and Environmental Scientific Research. 159: 450-45

*Corresponding author.

E-mail address: Workinehlemma6@ gmail.com/alemayehuredi5@ gmail.com 